(C)2009 IEEE. Personal use of this material is permitted. However, permission to reprint/republish this material for advertising or promotional purposes or for creating new collective works for resale or redistribution to servers or lists, or to reuse any copyrighted component of this work in other works must be obtained from the IEEE. 


\title{
Travel Aids For The Blind - The Digital Ecosystem Solution
}

\author{
David J. Calder \\ Curtin University of Technology \\ Bentley, Perth, \\ West Australia \\ Tel. 61-8-9266 2875 \\ D.Calder@curtin.edu.au
}

\begin{abstract}
Travel aids for the blind are portable electronic devices that are either cane fitted, hand-held or worn by the visually impaired user, to warn of obstacles ahead. These devices exhibit a number of complex user challenges, the most significant of which are related to the interface display that conveys navigation/obstacle warning information. There is a need for a new approach to the design of computer-based assistive systems for the disabled. Many Travel Aid manufacturers have catered for particular disabilities in relative isolation and not necessarily within the wider context of other modalities and areas of disability. The (DESAT) approach would help a fragmented cottage industry by offering a coordinated digital ecosystem from which both the ecologically embedded end user and support teams from diverse disciplines could benefit.
\end{abstract}

Keywords -User Interface, DESAT, disabled, blind, visually impaired, sensory channels, ambient sound cues, cognition, assistive technology, sound interface displays, laser, infrared, ultrasonic pulse-echo, long cane, portable electronic device,

\section{INTRODUCTION}

$\mathrm{T}$ here are two broad categories of device which are used for navigation by the blind. The first is the mobility aid which warns of hazards and objects in the path of the user, the second being of the orientation type which is usually more complex and expensive and attempts to map the forward looking environment for the user. There are approximately ten competing mobility aids and orientation mapping devices for the blind on the market at present, some with significant drawbacks. Devices can be heavy and cumbersome, which is very problematic in a device intended for extended periods of use. Many of these devices are highly visible, advertising the user's disability.

Devices that appear to be advanced because they exploit the latest technology, may in fact be working against other operational user sensory channels, in the process of conveying spatial information. This adds up to a potentially dangerous situation for visually impaired users. It is important not to create a false sense of security in the user, whilst at the same time masking all-important alternative modalities the user is accustomed to. Because the visual input channel can no longer be used, the interfaces on these devices rely on loading the other senses. This is fine as long as the alternative channel does not itself already play a vital part in conveying spatial information to the brain. Designing systems for people with a visual disability, requires more than expertise in electronics and software. There is a requirement for a multidisciplinary development team, a coordinated effort, particularly with respect to user interface design.

What is needed in order to achieve the above, is a new dynamic approach, a co-operative support strategy to assist the present cottage industry in the design of travel aids for the blind. The Digital Ecosystems for Assistive Technologies (DESAT) is suggested as part of such a solution.

\section{INTERFACING OPTIONS}

One class of aids use vibrating sectors in the display to warn of upcoming obstacles, a method which may transfer limited information regarding direction and proximity of a frontally located potential hazard. However, these interfaces do not usually disrupt a vital channel such as hearing.

Alternatively, the more spatially instructive devices use an audio interface in order to transfer more complex data streams to the user. However, this automatically compromises the user's hearing, a serious and limiting factor for a blind user.

The situation is compounded if people have one or more progressive disabilities, including visual impairment. Such blind users are dynamically interactive within an ecology that is itself constantly adapting and changing in real time. The human system is constantly interacting with others as well as other machine systems within the overall ecology. As these human systems evolve, so the whole surrounding ecology evolves too. Design for the disabled has to be seen in this context.

Although many mobility aids and more specifically orientation aids can warn of obstacles up to six metres 
ahead and crudely convey the distance of objects to the client, they cannot convey what would normally be regarded as field of view information to the user without compromising other critical sensory channels. This is a key flaw with most complex substitution devices that make the use of a persons hearing in order to convey forward mapping information. Ambient sound cues are vital to a blind person. They should not be compromised in any way. Also, the deaf/blind can obviously make no use at all, of such a sound interface display!

GPS systems are obviously not stand-alone devices in the way others are. They have had some success in addressing forward mapping of the big picture. They also help the user in orientation, clearly identifying where he or she is in a wider spatial theatre. However, they seldom warn of obstacles immediately ahead in real-time, are often unsuited to indoor use, being heavy/bulky to wear, typically are expensive and often severely mask the user's auditory channel. They therefore cannot be regarded as independent, as with other methods. Reducing real-time GPS-generated spatial information to a series of synthetically converted speech directions, helps to some degree in orientation, but is no substitute for vision. Requiring an area of the brain devoted to speech to act as language interpreter for such complex 3 dimensional data loads the cognition of the user. Most old and frail people would find such an alternative impossible.

If the blind user's cognitive capability, already loaded with the interpretation of synthetic speech, is only in fact presented with limited close-range orientation feedback, confidence will plummet. Not only is quality of life impaired, the frail user in particular, will quickly tire. At this point the user will fall back on the simple Long Cane.

For many of the other portable devices, user interface consoles seem similar to Morse Code receivers. A user experience may in fact be punctuated by non-specific on/off warning signals from his or her mobility aid.

Blind people often prefer the Long Cane because of its inherent simplicity and predictability and the fact that the skills required for its use have already been well established in the mind of the user.

\section{SMALL SPINOUT COMPANIES}

The companies responsible for competing mobility devices are different to the mainstream. They often originate from an academic base, such as with the author's development. Another example would be Sound Foresight, a spin-out company from Leeds University in the UK.

There is no single company with a significant market advantage against the competition. The author's initial prototype is shown in Fig 1. This has become a test bed for improved designs which are in the pipeline. Although, even in its present form, it could possibly compete commercially with many other products, it also certainly suffers from some of their common failings.

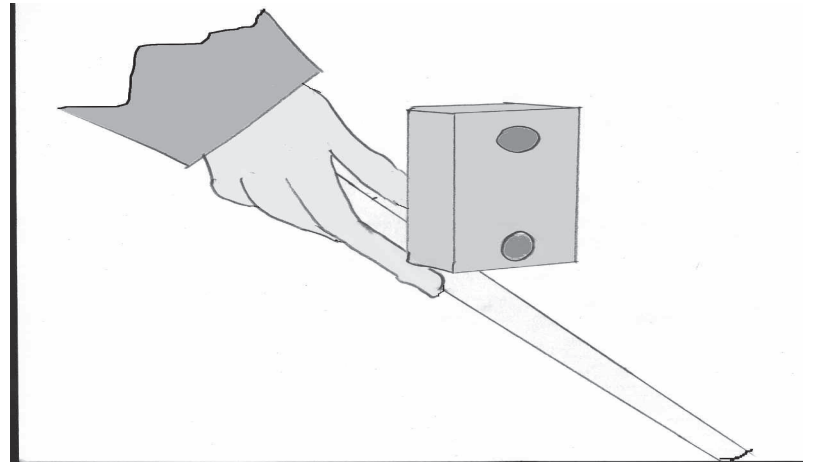

Fig. 1. Author's first prototype. Here, the ultrasonic transceiver circuit and user display are incorporated into the casing and attached to the handle of a functional long cane. The warning signals are displayed on a vibrating tactile interface.

A more ergonomic and integrated approach than that found on our initial prototype, would be to incorporate the sensors, ultrasonic transmitter, circuit, user display and battery into the cane handle (see Fig.2). The UltraCane does just this and feeds information about upcoming obstacles through to a series of vibrating buttons on the handle, conveying distance and rudimentary height information. It has two ranges, three metres and five/six metres, and its sensors detect from several centimeters off the ground to the top of the head. Since its launch in 2004, Sound Foresight has sold UltraCanes into around twenty countries. It has been featured on television programmes and in newspapers and magazines around the world, and won awards and design prizes.

The Tom Pouce is an infrared proximeter that can detect obstacles within a $0.5,1.5$ or 3 meters range. Users can select the desired range by means of a three-position switch. If an obstacle is in the chosen range a vibration is produced. At the $1.5 \mathrm{~m}$ setting, the coverage beam covers the vertical range from the knees to the head. However, infrared systems can be adversely affected by sunlight and are often limited to indoor use.

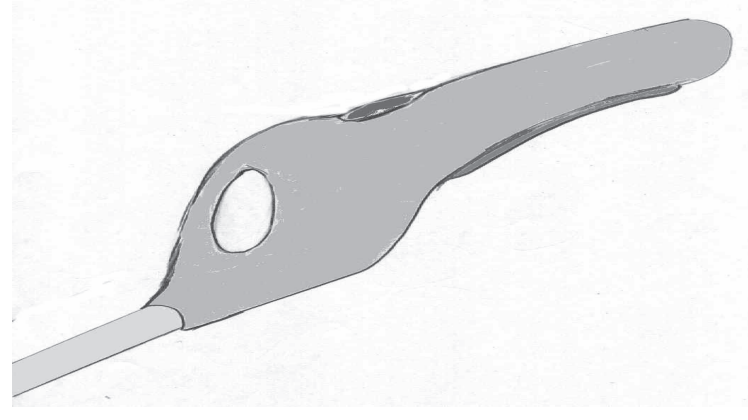

Fig. 2. An ergonomic solution. Using a tactile display the system represented is similar to the Ultracane. It also uses a timed return signal set, to locate obstacles in the path of the transmitted ultrasonic signal. The system is fully incorporated into the cane and handle. 
Smaller compact devices such as the one portrayed Fig 3, like the Miniguide, use ultrasonic echo-location to detect objects. The Miniguide vibrates to indicate its proximity/distance to objects - the faster the vibration rate the closer the object. There is also an earphone socket which can be used to provide sound feedback to the user. A single push button is used to switch the aid on or off and also change settings. The aid can accommodate ranges of between $0.5 \mathrm{~m}$ and $8 \mathrm{~m}$, depending on the chosen mode. The Miniguide has a transmitter/receiver pair that can be easily identified in the picture. Echo-location technologies typically operate in the $50,000 \mathrm{~Hz}$ region, well above the human audible limits of 20,000 Hz. They are therefore non invasive and use little battery power, which is important in keeping the portable device weight to a minimum.

\section{USER INDIFFERENCE TO TRAVEL AIDS}

Clear evidence of why current aids are rejected can be found in relevant conference and journal papers such as in The Journal of Visual Impairment and Blindness [1]. Blasch states that few of these assistive devices are regularly used. Davies in 2006 refers to only limited continued use of the device [2].

One of the sophisticated head-mounted devices, the Sonic Pathfinder is also referred to. The device has its sonar transducers mounted in an array in the front head band. The device is assessed on the basis that it is centerweighted, prioritizing obstacles immediately in front of the user, but perhaps not giving enough wide-angle coverage [3]. ETA rejection is listed in the report from National Research Council [4]. This report refers to auditory interfaces that compromise the natural feedback derived from tapping a long cane. Such auditory displays are still the most common interface in more sophisticated orientation devices.

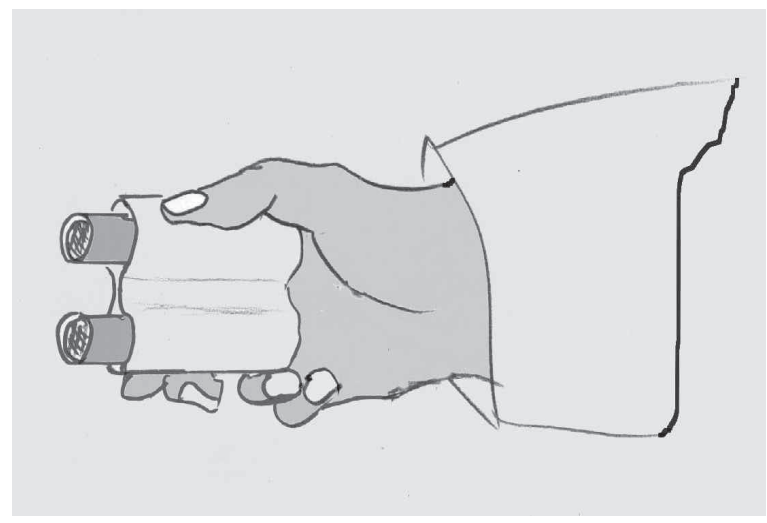

Fig. 3 A compact alternative such as that represented above, is the Miniguide, which has the advantage of a low current requirement. However, when used indoors, most of the ultrasonic devices pick up unwanted ambient echos from adjacent walls, ceilings and surfaces which may interfere with the interpreted processor result.

An early version of a relatively powerful laser system, the Teletact, made use of a laser only, the reflected beam of which, can result in a confused signal from plate glass, such as in a door or front to a building. There is also a problem with lasers not picking up black objects. Grass in front of the user could also compromise readings from a laser obstacle warning system.

The solution would appear to be to incorporate two methods in one, longer range laser and infra-red proximeter for short range transparent surface warnings where lasers don't work. In case of both proximeter and laser telemetric detection, the Teltact 2 system normally transmits telemeter information.

When the proximeter signal acitvates, it sends a glass warning output flag to the user, in order to warn that they may be approaching a window. The system operates within a range of 3 meters, and gives a window pane / black car detection up to two meters. Infra-red systems tend to be best suited to close range operation and are not immune from interference from the sun. There is no quick fix.

Although the Teletact 2 overcomes some of the problems associated with the previous model, it is has only a go/no go interface. It is essentially a binary switch interface (See Fig. 4).

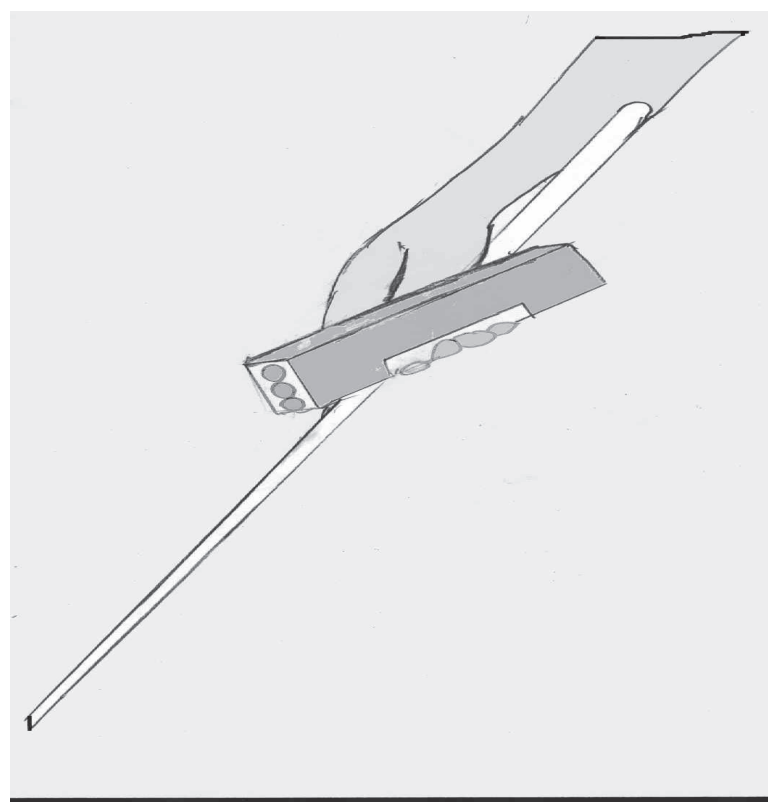

Fig 4. A solution such as that of the Teletact 2 is unique in that it makes use of both an infra-red proximeter and laser telemeter. Infra-red systems usually work well indoors, but can be adversely affected by interference from extraneous sources, such as sunlight.

The Teletact 2 exploits a vibrating display tactile communicators or tactors, located under the user's fingers. Tests were conducted with two, four and eight vibrating units, and the four-unit solution turned out to be the most appropriate. Each finger (except the thumb) is in contact with one tactor. Each tactor corresponds to a distance interval. If an obstacle is detected within one of the four distance intervals, then the corresponding tactor in the interface console is triggered. 


\section{AN AGING POPULATION}

Projections indicate that by 2024 , over 800,000 Australians will suffer from visual impairment, and approximately 90,000 will be blind [5]. The total number of Americans with blindness in 1995 was approximately 1.3 million, and that number grew to 1.5 million in 2000 . Incorporating the high death rates for older age groups, the expected net growth in the prevalence of low vision and blindness is approximately 36,000 cases per year until 2025. However, the annual incidence, the number of new cases added each year, will grow from the current 256,000 to 500,000 in 2020 [6].

In 2002, The World_Health Organization estimated that there were 161 million (about $2.6 \%$ of the world population) visually impaired people in the world, of whom 124 million (about 2\%) had low vision and 37 million (about $0.6 \%$ ) were blind [7].

Excluding China and India, in developing countries, 18.8 million people were blind in 1990 compared to 19.4 million in 2002, an increase of 3\%. In China and India the estimated numbers of blind people in 1990 were 6.7 and 8.9 million, respectively; in 2002 there were an estimated 6.9 million blind people in China and 6.7 million in India. These figures indicate an increase of $3 \%$ in the number of blind people in China and a decrease of $25 \%$ in India.

The following is a quote from Margrain [8]:

"The number of people with impaired sight that cannot be improved with the use of spectacles or other treatments is growing. Demographic data suggests that the numbers of people with impaired vision are likely to increase at least until 2021 because the main causes of low vision are age related. Medical intervention is unlikely to reduce significantly the numbers of people with impaired vision in the foreseeable future because there is currently no treatment for the primary cause of visual impairment, age related macular degeneration. Given that it will not be possible to cure visual impairment the emphasis must be on providing an effective rehabilitative low vision service."

Statistics from the Canadian National Institute for the Blind (CNIB) show an increase in those in need of services from their organization; and these numbers are considered to be conservative because data collection is a result of self-report and collected from individuals who participate in their services.

Eighteen percent of hip fractures by older Americans are caused by vision impairment. The cost of treatment is $\$ 2.2$ billion per year. It is estimated that US\$441 million would be saved annually, if support systems could prevent just one fifth of these fractures [9].

\section{DEVICE DESIGN CRITERIA}

Devices available in the market can be broken down into three categories. First the simpler type that warn of an obstacle in the forward vicinity of the user, but convey little or no detail with respect to position or object identification. They may use buzzers, simple warning vibration or synthetic tones as the user interface. They do not warn of drop-offs, such as potholes, in any truly reliable way.
The second category may have enhanced range and precision, as in the case of some laser based types, but often these have a far too simplistic binary information go/no go user interface. Complex orientation systems (third category) may use sonar sweeping techniques that convert ultrasonic reflected signals into a stretched synthetic but inhuman audio frequency that is presented to the user. Such devices require substantial learning and often compromise the natural sound cues that are absolutely essential for a blind person.

Many of the competing products have poor and inappropriate human-machine interfaces, such as head mounted displays. A recent paper in the Proceedings of the 2005 IEEE Engineering in Medicine and Biology Conference reinforces these views [10]. Velazquez et al confirm that, although many ETAs have been proposed to improve mobility and safety navigation independence for the visually impaired, none of these devices is widely used and user acceptance is low.

Hakkinen's IEEE conference paper [11] refers directly in the title to 'Postural Stability and Sickness Symptoms After Head Mounted Display Use.' The findings show clearly these common displays produce adverse affects on the user.

\section{USING SOUND IN THE DISPLAY}

With some of the more sophisticated systems, the display consists of a stereo montage of synthetically generated sounds which represent the layout in front of the user. In one version, scanned objects produce multiple echoes in the receiver, which are translated by the processor into unique invariant 'tone-complex' sounds, which users listen to and learn to recognize.

Beyond the safety aspect, blind users have learned to depend on their hearing, and any product which continuously interferes with it may lead to a compromised alternative human sensory input, and therefore quality of life including social and psychological aspects. A specific reference can be found from Johnson and Higgins who refer to visual-auditory substitution taxing a sensory modality that is already extensively used for communication and localization [12]. A Study by Ross and Blasch [13] clearly indicated that blind people preferred a tapping tactile interface to sound feedback.

Head mounted displays usually incorporate sound feedback which has already been covered. However, they may be bulky, relatively heavy and consequently are often rejected by the user [10 and 11]. Velazquez refers to them as being invasive and disturbing the environment [10]. The author's own view is that feedback and scanning technologies are in fact quite acceptable, as long as the design is carefully initiated from the user's perspective and requirement first, with an emphasis on minimizing the loading of human cognition and alternative sensory pathways. Unfortunately and typically, it is the front-end scanning electronics which are designed first and the user interface (which is a complex bottleneck) that is considered later in the design. This user bottleneck is commonly the biggest problem with these devices. 


\section{TECHNOLOGY DRIVEN SOLUTIONS}

Many designs for disabled users fall short as ongoing adaptive solutions. In practice, it is currently almost impossible to offer an assistive technology that will match and then continue to match the user requirements of an ever evolving ecosystem, as is the case where human input and output channels and the complexities of human-tohuman and human-to-machine communication are concerned. The rejection rate of currently produced assistive devices reinforces this claim. Each individual is a moving target for the system designer, as the day-to-day progression or learning rate for each user is different. Dynamic interactive solutions are required.

There are also factors such as the time and financial investment involved in designing and manufacturing for the individual in an isolated vacuum. The answer is often a compromise between these parameters that is only partially successful.

The challenges are compounded if the user has multiple disabilities [14]. The design team, who may be focusing exclusively on one area of disability, does not always consider these multi-facetted demands.

Multiple disabilities can be physical, cognitive or both. Issues of complexity with respect to individual requirements must be seen within the context of a wider ecology of the particular user, with that person clearly at the centre, contributing to a team solution. An established and highly successful ecological approach to designing individualized education programmes for the disabled student has been refined over twenty years into a highly recommended model and is now regarded as 'best practice' [15]. This ecological approach has not as yet permeated all areas of disability support. However, the power of the digital ecosystem framework is now accepted within many other disciplines, particularly with respect to small enterprise collaboration [16].

Within small business, the advent of the web has allowed sales penetration over vast distances. Accompanying these advances have come new modes of marketing and partnership possibilities that would have been impossible only a few years ago. With this connectivity has come a fertile and dynamic business theatre that cannot be avoided if small enterprises are to survive. This interaction has led to collaborative workflow models [17]. The logic behind collaborative workflows is to produce a sequence of activities that not only produce a meaningful result, but also to facilitate small groups working together to achieve common goals. The actual physical distance and associated limitations between these entities then becomes less important as web-based tools are used to link enterprises and their common aspirations [18]. The entities themselves may be small companies competing against large predator corporations, or widely dispersed cottage industries (such as those associated with assistive devices and the manufacture of travel aids) with a common interest [19].

\section{EARLY PROTOTYPE SOLUTIONS}

The author has designed and built an ultrasonic frontend linked to a unique interface. The device has been tested when fitted on a standard cane, but is not limited to this form of use. In its basic first prototype form, this design has been ideal for testing various interface options. From this feedback, further designs are now being built, with the emphasis on ease of learning, proving simple interface options, and a very close examination of the alternative sensory channels. Assuming multiple disabilities in the user is, and has been, at the forefront of the design requirement.

It is hoped that the results from work up to this point and over the next few years will allow us publish in some more comprehensive detail. However, after twenty years in the general area of assistive technology design for the disabled, the author is hopefully realistic and not claiming to be able to offer a substitution for sight.

\section{DESAT AS A SOLUTION}

Digital Ecosystems for Assistive Technologies (DESAT) is a collaborative cluster-based ecosystem, neither limited by distance between clusters nor the particular disability types associated with each of the clusters. Individual clusters may include a range of specialist personnel associated with the support of a client requirement. The output of such an environment would not only be the efficient research and development of appropriate assistive devices, but also result in more streamlining for the teams in their everyday support of an individual, such as training in the use of a long cane or mobility aid for the visually impaired.

With each client representing a nucleus at the centre of his or her support cluster, an individual's local ecological environment has been acknowledged (as discussed and cited in previous sections) as a worthwhile starting point, offering a framework from which specialist support action may be fleshed out.

Each support cluster would have a number of clients within its particular category of disability. Cluster membership would not be determined by distance or physical boundaries. The aim would be to maximize use of the digital ecosystem paradigm in order to break existing physical boundaries.

The protection of intellectual property (IP) would remain uppermost as one of an individual company's prime commercial considerations. The difference would be in the focus and modular consideration of appropriate novel and relevant ideas, when initially considering I.P. matters.

\section{DESAT STRUCTURE}

A cluster of people with a vast range of interdisciplinary skills would focus on a user group of people, all with a common disability. There would be many separate clusters, meeting the challenges and specific needs of different disability groups. As now, it may be assumed that special education specialists, therapists, medics, 
academics, engineers and particularly hardware and software experts would form part of each cluster, the main difference being a recognition of the greater ecosystem in which each cluster coexists and operates.

Users at the center of each cluster, the nucleus, would determine the nature of the environment. Clusters would communicate with each other for a common good and the ecosystem itself would be able to benefit from its size in terms of external links and its critical mass.

\section{CONCLUSION}

In many respects, Travel Aids for the blind have not been widely accepted by those who are desperately seeking a viable solution to loss of vision. As most commercially available travel aid devices are produced by small, unlisted companies, there is little in the way of publicly available, reliable sales figures, and as such the addressable market is not well defined. However, literature searches, interviews conducted with industry experts, in addition to the small size of the companies themselves, indicate clearly that these competing devices have so far failed to achieve any significant market presence or widespread user acceptance.

A Digital Ecosystem For Assistive Technologies (DESAT) model has been proposed in order to formulate methods aimed at addressing the above problems. The ultimate aim of DESAT would be improving the quality of life for people with a range of disabilities, with particular reference to the blind client in the case of this particular paper.

The DESAT ecosystem is made up of dynamically changing entities, client-support clusters being the most important. Clusters are client-centred, with an interactive group representing the nucleus and contributing to a particular cluster direction.

A global advantage and DESAT's greater mass would benefit small assistive technology companies and the wider ecology on many levels. There would be lower manufacturing costs than is now associated with small-run dedicated systems production. This advantage would result from greater demand for DESAT modular units across clusters and existing boundaries. Relatively large production runs catering for a global DESAT module demand would drive production costs down.

By applying a DESAT strategy, current digital technologies such as mobile, the internet and video conferencing could be coordinated and optimised to deliver the best outcome for all members of this support ecosystem for the blind and also for small spin-out assistive technology companies, as is the case in this particular context.

\section{REFERENCES}

[1] B. Blasch, results of A National Survey of Electronic Travel Aid Use. Journal of Visual Impairment and Blindness 83, pp 449-453. 1999.

[2] T. Davies, C. Burns and S Pinder, "Using Ecological Interface Design to Develop an Auditory Interface for Visually Impaired travelers," Proc. Of OZCHI 2006, Sydney, Australia. 2006

[3] K Young-Jip, K. Chong-Hui and K. Byung-Kook, " Design of Auditory Guidance System For The Blind With Signal Transformation from Stereo Ultrasonic to Binaural Sound," Proc of $32^{\text {nd }}$ ISR (International symposium on Robotics), April 2001.
[4] Committee on Vision "Electronic Travel Aids: New Directions For research," Working group on Mobility Aids For The Blind, National research Council, pp 74, National Academy press, Washington, DC 1986.

[5] Vision 2020: the Right to Sight 1999 - 2005 p.19

[6] Bulletin of the World Health Organization, p.847, Nov. 20

[7] World Health Organization: Magnitude and causes of visual impairment, p.3, Nov 2004.

[8] Margrain, TH. (2000). Helping blind and partially sighted people to read: the effectiveness of low vision aids. British Journal of Ophthalmology. 84, 919-921.

[9] Vision Rehabilitation: Evidence-Based Review, p.25, May 2005.

[10] Velazquez, E. Pissaloux and F. Maingreaud, "Walking Using Touch", Proc. Of 2005 IEEE Engineering in Medicine and Biology $27^{\text {th }}$ Annual Conference, Shanghai, China, 2005.

[11] J. Hakkinen, "Postural Stability and Sickness symptoms After HMD Use", Proc. Of IEEE International conference on Systems, Man and cybernetics, Hammamet, Tunisia, 2002.

[12] L Johnson and C Higgins, "A Navigation Aid for the Blind using Tactile-Visual Sensory Substitution," Department of Electrical and Computer engineering program, university of Arizona, Tucson USA. 2006.

[13] D. Ross and B. Blasch, "Wearable Interfaces for orientation and wayfinding," Proc. Of ASSETS 2000, Arlington, Virginia, USA. 2000.

[14] J. Jacko, H. Bautsch, "A Conceptual Framework For The Integration of Clinical Diagnosis, Functional Capabilities and Classes of Technology", University of Wisconsin Lab for HumanComputer Interaction. Technical Report series no. 1131, 2000, pp. $1-59$

[15] B. Rainforth, J. York, C. Macdonald, Collaborative Teams for Students With Severe Disabilities, Baltimore: Paul Brookes, 1993, pp. 71- 83.

[16] E. Chang, M. West. "Digital Ecosystems and Comparison to Collaboration Environment". WSEAS Transactions on Environment and development 2, 2006, pp. 1396-1404

[17] L. Pudhota, Chang E, "Modelling the Dynamic Relationships between Workflow Components" ICEISI Porto, Portugal, 2004.

[18] D. Neumann," An Introduction to Web Objects". On-line overrview. 2004

[19] M. UlieruR. Brennan Scott, "The Holonic enterprise: a model for Internet-enabled Global Manufacturing Supply Chain and workflow Management”, Canada, 2000 